\title{
Actualización en el diagnóstico y manejo de la pancreatitis aguda
}

\section{Update on the diagnosis and management of acu- te pancreatitis}

Pág. 51,62

Recibido: $17-06-2020$

Aceptado: 20-08-2020

Dra. Rebeca Gutiérrez Navarro ${ }^{1}$

Dr. Gabriel Alexis Sanabria Cordero ${ }^{2}$

1. Investigadora independiente, Cartago Costa Rica.

2. Consultorio Médico Dr. Obando,Investigador independiente, Cartago Costa Rica.

\section{RESUMEN}

La pancreatitis aguda es un proceso inflamatorio que puede limitarse al páncreas o puede extenderse a estructuras vecinas, provocando así, un síndrome de respuesta inflamatorio agudo que puede culminar en la muerte. Su síntoma pivote es el dolor abdominal agudo y su diagnóstico se basa en tres pilares: clínica, analítica e imágenes.

\section{PALABRAS CLAVE}

pancreatitis aguda, dolor abdominal, fallo multiorgánico, páncreas, enzimas pancreáticas

\section{ABSTRACT:}

Acute pancreatitis is an inflammatory process that can be limited to the pancreas itself, or extended throughout neighbor structures, causing an acute inflammatory response syndrome that can lead to death. Its pivotal symptom is acute abdominal pain, and its diagnosis is based on three main pilars, which are: clinical signs and symptoms, laboratory findings, and imaging.

\section{KEY WORDS}

acute pancreatitis, abdominal pain, multiorgan failure, pancreas, pancreatic enzymes.

\section{INTRODUCCIÓN}

La pancreatitis aguda es una patología inflamatoria, de presentación variable, que puede ser edematosa, hemorrágica o necrótica. Actualmente, cuenta con una alta incidencia en servicios de emergencia a nivel global y ha aumentado de forma paulatina, entre 4,9 y 73,4 casos por cada 100.000 habitantes; a nivel mundial, el $20 \%$ suele tener un curso más 
tórpido, que de no ser abordado precozmente condiciona al desarrollo de complicaciones locales, evoluciona a disfunción multiorgánica y aumenta la mortalidad en un $10-30 \%$ del total de casos severos (1). En los últimos años, se han desarrollado cambios con respecto al abordaje y manejo médico y quirúrgico, así como de sus complicaciones tanto locales como sistémicas; sin embargo, muchos autores reconocen que existe cierta controversia en torno al manejo más apropiado de las distintas circunstancias clínicas con las que se presenta esta patología.

\section{DEFINICIÓN 4380989505574612}

Se define como una lesión inflamatoria local del parénquima pancreático asociado o no a la inflamación sistémica, que puede llevar a compromiso y disfunción multiorgánica. Se debe a la liberación y activación inadecuadas de las enzimas pancreáticas que destruyen el tejido pancreático $\mathrm{y}$, por consiguiente, se desarrolla una reacción inflamatoria aguda.

La clasificación de Atlanta (revisada, 2012), proporciona la terminología más aceptada para clasificar los tipos morfológicos y clínicos entre diferentes especialistas y expertos para llegar a un consenso global incorporando la evolución de los estudios científicos realizados durante 20 años. Esta clasifica la pancreatitis según su severidad como (i) pancreatitis aguda leve, caracterizada por ausencia tanto de necrosis peripancreática como de fallo orgánico; (ii) pancreatitis aguda moderada, caracterizada por cualquier tipo de necrosis pancreática estéril o fallo orgánico transitorio*; (iii) pancreatitis aguda grave, cualquier tipo de necrosis pancreática infectada 0 fallo orgánico persistente ${ }^{* *}$ y (iv) pancreatitis aguda crítica; cualquier tipo de necrosis pancreática infectada y fallo orgánico persistente (2). Los criterios de Atlanta definen fallo orgánico con $\geq 2$ puntos.

*Fallo orgánico transitorio: resuelve en 48 horas después el abordaje adecuado.

**Fallo orgánico: no resuelve en 48 horas después del abordaje adecuado.

\section{Las Guías del Colegio Americano de Gastroenterología describe dos fases de la pancreatitis aguda (Tabla 1.)}

\begin{tabular}{|c|c|}
\hline $\begin{array}{c}\text { Pancreatitis } \\
\text { aguda temprana }\end{array}$ & $\begin{array}{c}\text { Pancreatitis } \\
\text { aguda tardia }\end{array}$ \\
\hline$<1$ semana & $>1$ semana \\
\hline $\begin{array}{c}\text { Respuesta } \\
\text { inflamatoria } \\
\text { sistémica }\end{array}$ & $\begin{array}{c}\text { Complicaciones } \\
\text { locales }\end{array}$ \\
\hline $\begin{array}{c}\text { Fallo } \\
\text { multiorgánico }\end{array}$ & \\
\hline
\end{tabular}

Tabla 1. Fases de la pancreatitis aguda.

\section{EPIDEMIOLOGÍA}

La incidencia de pancreatitis aguda (PA) es variable y ha aumentado de forma paulatina globalmente, entre 4,9 y 73,4 casos por cada 100.000 habitantes a nivel mundial. En Latinoamérica, se reportó en el 2006, una incidencia de 15,9 casos por cada 100.000 habitantes en Brasil, una prevalencia del 3\% en México en el 2014 y, en Perú, refieren una incidencia de pancreatitis de 28 casos por cada 100.000 habitantes en el 2009 (3). Ha habido aumentos importantes en la incidencia, sobre todo de población pediátrica, ese riesgo 
incrementado de pancreatitis se relaciona estrechamente con la epidemia mundial de obesidad y con el aumento de tasas de incidencia de colelitiasis. (4).

Alrededor de un $80 \%$ de los pacientes se recupera totalmente en 1 semana, desarrollando enfermedad leve no complicada, sin sepsis y sin requerir tratamiento de forma intensiva. Sin embargo, alrededor de $20 \%$ de los pacientes presenta complicaciones tanto locales como sistémicas, esos pacientes que cursan con complicaciones presentan una tasa de mortalidad a su vez de 10\%-30\% (5). Ante la presencia de síndrome compartimental abdominal, las fatalidades pueden llegar al $49 \%$ (6). La mortalidad relacionada con la pancreatitis, no obstante, ha disminuido, siendo esta de un $2 \%$ a nivel global (4). Dentro de las causas más frecuentes se encuentra la litiasis biliar $(40 \%)$, alcohol $(30 \%)$, hipertrigliceridemia $(2-5 \%)$, drogas $(<5 \%)$, CPRE $(5 \%)$, entre otras. (4)

Según el Instituto Nacional de Estadistica y Censo para Costa Rica (INEC), en el año 2019 se documentaron 49 muertes atribuibles a pancreatitis aguda.

\section{ETIOPATOGENIA}

En condiciones normales el páncreas exocrino secreta enzimas de forma en gránulos de cimógeno inactivos que protegen a la glándula de autodigestión. La tripsina se sintetiza en proenzima inactiva, y cuando ocurre una activación intrapancreática de esta, puede provocar la activación de otras proenzimas como la profosfolipasa y proelastasa que degradan células adiposas y lesionan fibras elásticas de los vasos sanguíneos, respectivamente. La consecuente inflamación y trombosis de los pequeños vasos lesionan las células acinares, amplificando la activación de las enzimas digestivas (7).

En la tabla 2 se resume la etiología de la pancreatitis. Dentro de los mecanismos propuestos de la patogénesis, se menciona la obstrucción del conducto pancreático, incrementa la presión ductal intrapancreática y esto conduce a la acumulación de líquido rico en enzimas, como cimógenos inactivos en el intersticio, sin embargo, la lipasa se secreta en forma activa y conduce a una necrosis grasa local. La muerte de adipocitos genera señales de peligro a nivel local que estimulan la liberación de citosinas pro inflamatorias y otros mediadores de inflamación que inician un proceso inflamatorio local y fomentan el desarrollo de edema intersticial.

El edema compromete todavía más el flujo local de sangre, provocando lesiones isquémicas en células acinares (7). Otro mecanismo propuesto es la lesión primaria de células acinares, donde el aumento de calcio sérico juega un papel importante, como estímulo para la activación inadecuada de las enzimas digestivas. Fisiológicamente, actúa en la regulación de la actividad de la tripsina cuando disminuye el calcio sérico, produciendo degradación de la tripsina e inactivación, pero cuando aumenta el calcio, esta autoinhibición se anula y se convierte en su forma activa. El alcohol parece causar pancreatitis aguda por los mecanismos antes mencionados. Se cree que aumenta de forma transitoria la contracción del esfínter de Oddi. Tiene efectos tóxicos directos sobre las células acinares, oxidación de lípidos de la membrana basal y producción de radicales libres. 
El estrés oxidativo puede fomentar la difusión de los lisosomas y los gránulos de cimógeno y, alterar las concentraciones de calcio.

Obstrucción mecánica del conducto pancreático/ parénquima pancreático Cálculos biliares de colesterol, calcio Parásitos: Ascaris lumbricoides $y$ Cloronorchis sinensis $\left({ }^{7}\right)$ Lesiones latrogénicas: lesiones quirúrgicas, técnicas endoscópicas (CPRE)

Traumatismos

Metabólicos

Alcohol

Hipertrigliceridemia en $\geq 1,000 \mathrm{mg} / \mathrm{DI}$

Hipercalcemia

Fármacos: mercaptopurina, mesalazina, metronidazol, codeína, enalapril, isoniazida, simvastatina, ácido valproico, terapia antirretroviral altamente activa (HAART) $\left({ }^{2}\right)$.

\section{Genéticos}

Mutaciones en los genes que codifican la tripsina

\section{Vasculares}

\section{Shock}

\section{Vasculitis}

\section{Neoplasias}

Tabla 2. Etiología de la pancreatitis aguda

\section{CLÍNICA}

El dolor abdominal es la primera manifestación clínica. Se presenta como un dolor abdominal epigástrico de características intensas, constante, suele ser descrito como quemante y que usualmente se irradia "en banda" o "en cinturón" hacia la parte alta de la espalda $y$ al hombro izquierdo; sin embargo, no es específico. En ocasiones, se presenta como dolor abdominal generalizado. La fiebre está presente en la mayoría de los casos, debido a la liberación masiva de citoquinas y en presencia del síndrome de respuesta inflamatoria sistémica (SIRS). La anorexia, náuseas y vómitos suelen ser acompañantes típicos del cuadro agudo.

La pancreatitis severa, previamente descrita, representa el $15-20 \%$ de los casos (8). Existen diferentes métodos para evaluar la severidad del cuadro agudo, entre ellos los criterios de APACHE (completados en cualquier momento de la evolución) y los criterios de Ranson, los cuales se completan a las 48 horas de la admisión del paciente. Sin embargo, el mejor predictor es la escala BISAP, con sensibilidad y especificidad del $72 \%$ y $99 \%$ respectivamente (2).

Entre los factores asociados al agravamiento del cuadro, se destaca la edad > 55 años, comorbilidades asociadas, obesidad IMC $>30$ $\mathrm{kg} / \mathrm{m} 2$ y consumo crónico de excesiva cantidad de alcohol. (4)

Las manifestaciones clínicas iniciales varían en cuanto a su presentación, pudiéndose presentar como un cuadro leve de dolor abdominal que generalmente es autolimitado, hasta un síndrome de respuesta inflamatoria sistémica acompañado por taquicardia, disnea, taquipnea $y$ fiebre. 
En casos de pancreatitis necrótica, siendo esta presentación inicial de la patología o como complicación, puede hallarse en la exploración física, los signos de Grey Turner, que se manifiestan como coloraciones violáceas en ambos flancos o en formación periumbilical, conocido como el signo de Cullen.

El diagnóstico preciso de pancreatitis requiere dos de las siguientes tres características: dolor abdominal consistente con pancreatitis aguda, niveles de amilasa o lipasa sérica al menos 3 veces por arriba de su límite normal y hallazgos compatibles con pancreatitis en estudios de imagen, sea esta una TC o una Resonancia Magnética. (4). La elevación de la amilasa se produce a partir las 6-10 horas del inicio del dolor y en presentaciones no complicadas regresa a la normalidad en 3-5 días. Tiene una sensibilidad cercana al $70 \%$, sin embargo, su valor puede ser normal en un $50 \%$ de las pancreatitis agudas por hipertrigliceridemia y hasta un $20 \%$ de las pancreatitis agudas alcohólicas. (9). La elevación de la lipasa es más precoz que la de la amilasa, se produce a las 4-8 horas desde el inicio del dolor y permanece elevada de 8-14 días. Tiene una sensibilidad en rangos de $85 \%$ al $100 \%$, por lo que es superior frente a la amilasa, sobre todo en pacientes con poco tiempo de evolución de síntomas. (9) El grado de elevación de la amilasa o la lipasa no tiene valor pronóstico ni indica severidad. (9). El "gold standard" de estudios de imagen para valoración de la PA y sus complicaciones continúa siendo la TC, no obstante, su indicación es reservada, existen otras técnicas como el ultrasonido y la resonancia magnética (10).

La utilidad de la TC es limitada en pacientes que poseen alergias a medios de contraste IV, o que padecen de enfermedad renal. (11).
La principal indicación del ultrasonido abdominal es la valoración de la vía biliar en busca de colelitiasis y, para ello se considera el "gold standard". (9) Se recomienda realizar en todos los casos de pancreatitis en el contexto agudo dentro de las primeras 24-48 horas (12). La TC con medio de contraste o resonancia magnética se debe reservar para los siguientes contextos, los pacientes con diagnóstico incierto o como parte de la evaluación de diagnósticos diferenciales que lo ameriten, los que no mejoran o presentan deterioro clínico tras 48-72 horas de admisión hospitalaria, o que presente signos de sepsis que hagan sospechar infección de colección necrótica aguda. (6). Pacientes con síntomas abdominales no concordantes y una amilasa o lipasa sanguínea discretamente elevada no deberían recibir un diagnóstico de pancreatitis aguda o crónica. (4).

El tripsinógeno-2 en orina es otra herramienta diagnóstica, ha sido utilizado desde 1996 para su diagnóstico, con una sensibilidad entre $68-94 \%$ y una especificidad de $87-97 \%$, con un alto valor predictivo negativo (93.3\%), interpretación fácil, rápida e inequívoca y su utilidad radica en centros de salud que no dispongan de un laboratorio clínico. El tripsinógeno-2 no es un marcador de severidad (13). Dentro de la fisiopatología de la pancreatitis aguda existe una fuga capilar importante que puede conducir al paciente a una disminución del volumen circulatorio efectivo, hemoconcentración y shock; por lo que adquiere especial importancia la correcta fluidoterapia para alcanzar parámetros hemodinámicos adecuados y acordes con el paciente. (6). Los pilares del manejo de la pancreatitis contemplan un adecuado diagnóstico, un triage apropiado (evaluar severidad), soporte de alta calidad, monitoreo, tratamiento de complicaciones y prevención de recaída. (4). Los predictores de mayor severidad de Pancreatitis dentro de las

\section{(c) $(1) \Theta \Theta$}




\begin{tabular}{|c|c|}
\hline \multicolumn{2}{|r|}{ BISAP } \\
\hline 1. & $\mathrm{BUN}>25 \mathrm{mg} / \mathrm{Dl}$ \\
\hline 2. & Escala de Glasgow $<15$ \\
\hline 3. & $\begin{array}{l}\text { SIRS definido como } 2 \text { o más de } \\
\text { los siguientes }\end{array}$ \\
\hline $\mathbf{a}$ & $\begin{array}{l}\text { Temperatura }>\text { a } 380<\text { a } 36 \\
\text { grados Celsius }\end{array}$ \\
\hline $\mathbf{b}$ & $\begin{array}{l}\text { Frecuencia Respiratoria }>\text { a } 20 \\
\text { por min, o } \mathrm{PaCo} 2<32 \mathrm{mmHg}\end{array}$ \\
\hline c & Frecuencia Cardíaca $>90$ por min \\
\hline d & $\begin{array}{l}\text { Leucocitos }>12,000 \text { o }<\text { a } 4,000 \\
\text { cel } / \mathrm{mm}^{3} \text { o reticulocitos }>10 \%\end{array}$ \\
\hline 4. & Derrame pleural \\
\hline 5. & Edad $>60$ años \\
\hline
\end{tabular}

Tabla 3. Escala BISAP.

Para la reanimación con fluidoterapia, las guías del Colegio Americano de Gastroenterología recomiendan su administración de manera vigorosa, con $250-500 \mathrm{~mL} / \mathrm{h}$ durante las primeras 12-24 horas (6), o de 5 a $10 \mathrm{~mL} / \mathrm{kg} /$ hora, lo cual usualmente asciende de 2500 a $4000 \mathrm{~mL}$ en las primeras 24 horas. (4). Un estudio, por parte de Wu BU, Hwang JQ, Gardner TH, et al, elaborado en 2011, demostró la superioridad del Lactato de Ringer sobre Cloruro de Sodio al $0.9 \%$ en reducción de marcadores inflamatorios, sin embargo, según el Acta Gastroenterológica Latinoamericana (2019), en cuanto a evolución final de la enfermedad no se ha podido demostrar la supremacía del Ringer Lactato sobre otras soluciones cristaloides. El mal empleo de fluidoterapia, de forma excesiva, puede llevar al paciente a una hemodilución, la cual aumenta el APACHE, la necesidad de VMA, el síndrome compartimental, la aparición más temprana de infección, lesión renal aguda y más del doble de mortalidad, en especial si el hematocrito está por debajo de 35\%. (6).

Un análisis retrospectivo determinó que el exceso de soluciones $>3.056 \mathrm{~mL} / \mathrm{kg} / \mathrm{h}$ se asoció a mayor edema agudo pulmonar, hemodilución, empleo de diuréticos y mayor estancia intrahospitalaria; dicho análisis recomienda un parámetro seguro de 1.95 a $3.056 \mathrm{~mL} / \mathrm{kg} /$ hora en las primeras 48 horas. (14). Durante este tiempo (12-24 horas), una adecuada monitorización es indispensable, para evaluar la respuesta a la fluidoterapia con objetivos de PAM entre $65-85 \mathrm{mmHg}$, una frecuencia cardíaca a menos de 120 latidos por minuto y una diuresis de $0.5-1 \mathrm{cc} / \mathrm{kg} / \mathrm{hora}$. (9). Un punto a tener en cuenta es que, pese a la fluidoterapia, mientras el proceso fisiopatológico siga activo, el líquido suministrado va a tender a "fugarse", por lo que empeorará el cuadro y su evolución. (6). El dolor es el síntoma predominante en la pancreatitis aguda y debe ser adecuadamente tratado, con evaluación frecuente (9).

En diversos estudios se ha utilizado paracetamol, AINES y opiáceos, dentro de rango de seguridad, sin embargo, es importante individualizar al paciente para proveer una adecuada analgesia; con frecuencia se sobre utiliza opiáceos y bromuro de hioscina de forma inadecuada, mientras que los AINE y paracetamol se suele emplear en dosis subóptimas (6). Los opiáceos pueden ser una opción apropiada para el tratamiento del dolor en pancreatitis aguda.

Comparados con otras opciones analgésicas, pueden reducir la necesidad de analgesia complementaria. El opioide más extendido en el uso clínico es la morfina (la dosis recomendada es de $0,15 \mathrm{mg} / \mathrm{kg}+0,3-0,5 \mathrm{mg} / \mathrm{kg} /$ día. Rescates: $0,03 \mathrm{mg} / \mathrm{kg} /$ dosis), y existen alternativas como meperidina, tramadol, etc. Ninguno de ellos ha demostrado mejores resultados. Con respecto 
a los AINE, se debe de tener precaución por las complicaciones renales que puedan tener los pacientes, Ibuprofeno 2400 mg/día, Diclofenaco $150 \mathrm{mg} /$ día y Ketorolaco $90 \mathrm{mg} / \mathrm{día}$ son las dosis recomendadas. En el caso del ketorolac no debe utilizarse más de cinco días por el alto riesgo de complicaciones gastrointestinales. (2) El uso de bloqueo epidural es una opción en casos moderados y severos.

El bloqueo epidural se ha asociado con disminución de mortalidad, control de acidosis, mejora de microcirculación en lecho peripancreático, menor extensión de la necrosis, de vasodilatación esplácnica. (6). Con respecto a la nutrición, los pacientes con pancreatitis aguda leve deben reiniciar la alimentación enteral oral tan pronto como su situación clínica lo permita, con dieta sólida hipograsa y no suelen requerir soporte nutricional (9). Para ello, se recomienda realizar una evaluación nutricional dentro de las 24-48 horas de ingreso a la institución, para determinar la presencia y el grado de $\mathrm{MN}$ y permitir una intervención nutricional adecuada. No es necesaria la normalización de los niveles de enzimas pancreáticas ni la resolución total del dolor para reiniciar dieta, siempre y cuando haya ausencia de dolor severo, íleo severo, nauseas o vómitos. (6).

Con respecto a la vía de nutrición enteral, no existen claras diferencias entre la alimentación por sonda nasoyeyunal o sonda nasogástrica, no obstante, la sonda nasogástrica por su mayor disponibilidad y facilidad de colocación es más ventajosa (9). La nutrición parenteral debe ser utilizada como terapia de segunda línea, si la NE no es tolerada, cuando no se logra cubrir los requerimientos nutricionales o ante la presencia de íleo prolongado (2). El inicio temprano de dieta se asocia con disminución de mortalidad en un $64 \%$, y en un $61 \%$ el fallo multiorgánico; beneficio que es máximo a las 24 horas, sin embargo, se pierde si se inicia alimentación posterior a las 48 horas; no se recomienda de rutina el uso de probióticos, prebióticos, ni elementos traza (6). La mayoría de autores concuerdan que la antibioticoterapia en las primeras 72 horas, continúa siendo un tema controversial. En un ensayo clínico aleatorizado, se concluyó en reporte preliminar que, el uso de antibióticos profilácticos en la forma grave, no reduce las complicaciones infecciosas locales y/o sistémicas, necesidad de cuidados intensivos, ni impacta la mortalidad. Esta tendencia tendrá que demostrarse en reportes futuros (5). Únicamente se recomienda usar antibióticos en el caso de sospecha clínica o radiológica de infección de necrosis pancreática o colangitis aguda (9), si existe sospecha de necrosis (peri)pancreática infectada en las primeras 72 horas, se debe primero descartar otras causas de SIRS y/o de empeoramiento clínico, por su baja frecuencia en esta etapa; al descartar otras causas, se debe tomar muestras para hemocultivos, tinción de gran y antibiograma, (2).

Dentro de los estudios en curso, se encuentra un estudio piloto aleatorizado de Mayo Clinic, evaluando la eficacia y seguridad de Dabigatrán $150 \mathrm{mg}$ como tratamiento en la pancreatitis aguda, un anticoagulante que, además, es un fuerte inhibidor de la tripsina, la conclusión y los resultados de este estudio en curso se publicarán en el 2021. (15). Otro estudio en marcha es la evaluación de la seguridad y eficacia de Infliximab en pacientes con PA, estudio doble ciego placebo controlado que actualmente se encuentra en fase 2. Infliximab es un anticuerpo monoclonal que bloquea al factor de necrosis tumoral alfa (TNF-a) y que tiene participación importante en la patogénesis y la gravedad de la

\section{(9) $(1) \Theta$}


pancreatitis aguda; se estima que su conclusión y resultados se publiquen en abril del 2021 (15).

A nivel global, junto con las medidas expuestas anteriormente, se deben contemplar las pautas del manejo médico, como la nutrición enteral temprana, tromprofilaxis, profilaxis de sangrado digestivo, evaluar para síndrome compartimental, identificar disfunción orgánica y una vigilancia activa por infección.

\section{La colangiopancreatografía retrógrada} endoscópica (CPRE) con esfinterotomía, se emplea principalmente en pacientes con pancreatitis de etiología biliar, con una tasa de éxito superior al $90 \%$ y está indicada en aquellos pacientes que tienen evidencia de colangitis superpuesta en las primeras 24-72 horas; además, es una opción terapéutica razonable, en pacientes con coledocolitiasis documentada en imágenes o hallazgos clínicos altamente sugestivos de obstrucción biliar sostenida (ictericia, función hepática alterada, o vía biliar persistentemente dilatada) que condicionan a un empeoramiento clínico (4), empero, en estos se debe tratar de investigar la causa del incremento de enzimas hepáticas mediante otras técnicas de imagen menos cruentas como ecografía transabdominal, CPRM o USE antes de plantear la CPRE. (12)

Los pacientes con pancreatitis litiásica están expuestos a un mayor riesgo de recidiva y adquiere suma importancia elegir el momento idóneo para realizar la colecistectomía, ya sea durante el mismo internamiento o, después de la resolución del proceso inflamatorio en caso de pacientes que hayan tenido complicaciones pancreaticas locales (flemón grande que se extiende hasta el hilio hepático). (1)

La CPRE no tiene beneficio en PA leve, como prueba diagnóstica antes de colecistectomía, o en ausencia de las características mencionadas anteriormente (4). Con respecto a la colecistectomía en los casos de pancreatitis biliar, una revisión sistemática de 9 estudios con 998 pacientes con pancreatitis biliar, demostró que la colecistectomía temprana (en el mismo internamiento), se asocia con menor incidencia de admisiones recurrentes por pancreatitis, colecistitis y cólico biliar (6), además, la incidencia de pancreatitis recidivante puede ser de hasta el $60-80 \%$ en los meses siguientes (15). La colecistectomía laparoscópica, colangiografía intraoperatoria e instrumentación biliar laparoscópica es una estrategia que ha demostrado ser segura en pacientes con PA no severa (12). Los pacientes con un episodio grave de pancreatitis biliar deben ser sometidos a colecistectomía después de la resolución de la pancreatitis y las consiguientes complicaciones locales (15). Las complicaciones de la pancreatitis se clasifican en locales y sistémicas (tabla 3.), las cuales tienen diferentes tiempos de aparición en la evolución de la enfermedad.

\begin{tabular}{|c|c|}
\hline $\begin{array}{c}\text { Complicaciones } \\
\text { locales }\end{array}$ & $\begin{array}{l}\text { Complicaciones } \\
\text { sistémicas }\end{array}$ \\
\hline $\begin{array}{c}\text { Colecciones } \\
\text { líquidas } \\
\text { peripancreáticas }\end{array}$ & $\begin{array}{c}\text { Shock } \\
\text { hipovolémico }\end{array}$ \\
\hline $\begin{array}{c}\text { Necrosis } \\
\text { abłeAtipfonepdiganiengs } \\
\text { pancreática } \\
\text { (estériles o } \\
\text { infectadas) }\end{array}$ & $\begin{array}{l}\text { le la pancreatitis aguda } \\
\text { Falla renal }\end{array}$ \\
\hline Pseudoquistes & $\begin{array}{l}\text { Insuficiencia } \\
\text { respiratoria } \\
\text { (derrame pleural, } \\
\text { atelectasias, } \\
\text { síndrome distress } \\
\text { respiratorio del } \\
\text { adulto) }\end{array}$ \\
\hline
\end{tabular}


La pancreatitis necrótica aumenta la severidad y mortalidad. Esta constituye el $20 \%$ de los casos y su "método diagnóstico de oro", para esta y las demás complicaciones locales, es la tomografía axial computarizada (TC) con medio de contraste. Sin embargo, existen limitaciones a su uso, debido a que no debe realizarse antes de las primeras 72 horas. La necrosis puede existir como una o varias áreas necróticas focales o difusas en el parénquima que abarque $>30 \%$ o de $>3 \mathrm{~cm}$ de tamaño o en la grasa peripancreática. Puede ser estéril o infectada. Las guías de del Colegio Americano de Gastroenterología no recomiendan el uso de profilaxis antibiótica en necrosis estériles (recomendación alta, moderada cualidad de evidencia 8). Después de 7-10 días, la infección debe sospecharse si no existe mejora o aumenta el deterioro clínico del paciente. Deben iniciarse antibióticos profilácticos (recomendación alta, moderada evidencia8), posterior a la toma de cultivos necesarios, entre ellos, aspiración con aguja fina, guiada por TC. En cuanto a la presencia de infección, esta podría manejarse conservadoramente con antibióticos como quinolonas, carbapenémicos y metronidazol o por medio de drenajes percutáneos o de métodos más invasivos como el desbridamiento quirúrgico, siempre individualizando el contexto clínico del paciente.

Las colecciones líquidas peripancreáticas y los pseudoquistes suelen aparecen en las primeras 4 semanas posterior al inicio del cuadro de pancreatitis, de igual forma, pueden ser estériles o infectadas. Ambas complicaciones contienen material líquido y homogéneo. La diferencia clave entre ambas se debe a la pared bien definida que posee el pseudoquiste en contraste con la colección líquida. Suelen resolver espontáneamente, sin embargo, en ocasiones el drenaje del pseudoquiste es requerida. Sus indicaciones son: dolor persistente, obstrucción gástrica o duodenal, obstrucción biliar, ascitis, derrame pleural, aumento de tamaño progresivo durante el seguimiento imagenológico, signos de infección o sangrado, la posibilidad de malignidad quística pancreática y el tamaño $>6 \mathrm{~cm}$ (16). La primera línea terapéutica es el drenaje transmural guiado por endoscopia.

Los pseudo quistes persisten por la comunicación con el conducto pancreático principal o la estenosis distal del conducto. En estas situaciones debe evitarse el drenaje percutáneo por el riesgo de una fistula pancreática externa (1). La opción preferida es el drenaje interno guiado por US hacia el estomago, duodeno o la colocación transpapilar de una endoprótesis en el conducto pancreático.

El tratamiento de las complicaciones locales se basa en el estadío clínico, evolución del paciente y la respuesta deficiente al tratamiento conservador. Debe utilizarse vigilancia estrecha con la medición regular de marcadores inflamatorios como PCR y TAC con protocolo pancreático, si se sospechan complicaciones locales.

Algunos pacientes requieren cirugía mínima invasiva que no responden al tratamiento escalonado, como desbridamiento retroperitoneal con asistencia de video, necroseptomía transgástrica endoscópica $y$, en casos más severos en presencia de síndrome compartimental abdominal se quiere descompresión abdominal abierta. (1)

Entre otras complicaciones, la fístula pancreático entérica suele tener una incidencia del 3-10\% (17) en un periodo de 10-90 días posterior

\section{(ㅇ) $(1) \Theta \Theta$}


al cuadro de pancreatitis. Mayoritariamente se deben a necrosectomía. La rotura de un seudoquiste/quiste suele ser debido, bien a la cavidad abdominal o mediante una fístula hacia el tracto gastrointestinal, siendo el colon el órgano más frecuentemente afectado seguido del estómago y duodeno.

Debe tomarse en cuenta en pacientes que asocien vomitos, diarrea y hemorragia digestiva. El diagnóstico se realiza por medio de TAC con medio de contraste, a pesar de su menor sensibilidad en comparación con la CPRE. La presencia en una TAC convencional de aire en el interior de una zona de necrosis pancreática, puede indicar bien su infección o fistulización hacia tracto digestivo.

Cuando se documentan fístulas en el tracto digestivo superior, usualmente pueden abordarse de manera conservadora (dieta absoluta, antibióticos y soporte nutricional). En contraste, las fístulas dirigidas hacia el colon, raramente responden a medidas conservadoras, presentan una mortalidad del $17-67 \%$ y su tratamiento más frecuente es el quirúrgico, el cual se basa en 2 técnicas; diversión mediante ileostomía o colostomía y resección del tramo colónico afectado. (17)

En casos de falla orgánica: puede desarrollarse en cualquier etapa de la pancreatitis. Se relaciona con una respuesta proinflamatoria temprana o aparece de manera secundaria a complicaciones locales infecciosas. Por lo general se acompaña de SIRS tardía y síndrome de disfunción/falla multiorgánica.

La falla orgánica se califica con los sistemas de Marshall o SOFA. Los tres sistemas orgánicos más afectados con más frecuencia son

\begin{tabular}{|l|l|}
\hline Al ingreso & $\begin{array}{l}\text { Durante las } \\
\text { siguientes 48 } \\
\text { horas }\end{array}$ \\
\hline$>55$ años & $\begin{array}{l}\text { Disminución del } \\
\text { Hto }>10 \text { puntos }\end{array}$ \\
\hline $\begin{array}{l}\text { Leucocitos } \\
>16.000 \mathrm{m3}\end{array}$ & BUN $>5 \mathrm{mg} / \mathrm{dL}$ \\
\hline $\begin{array}{l}\text { Glicemia >200 } \\
\mathrm{mg} / \mathrm{dL}\end{array}$ & $\begin{array}{l}\text { Calcio sérico <8 } \\
\mathrm{mg} / \mathrm{dL}\end{array}$ \\
\hline $\begin{array}{l}\mathrm{LDH} \text { sérica }>350 \\
\mathrm{U} / \mathrm{L}\end{array}$ & $\mathrm{PO2}<60 \mathrm{mmHg}$ \\
\hline $\mathrm{AST}>250 \mathrm{U} / \mathrm{dL}$ & $\begin{array}{l}\text { Déficit de base }>4 \\
\text { meq/L }\end{array}$ \\
\hline & $\begin{array}{l}\text { Secuestro } \\
\text { estimado } \\
\text { líquido }>6 \mathrm{~L}\end{array}$ \\
\hline
\end{tabular}

Tabla 6. Signos pronósticos de Ranson alitiásica

\begin{tabular}{|c|c|}
\hline $\mathrm{Al}$ ingreso & $\begin{array}{c}\text { Durante las } \\
\text { siguientes } 48 \\
\text { horas }\end{array}$ \\
\hline$>70$ años & $\begin{array}{l}\text { Disminución del } \\
\text { Hto }>10 \text { puntos }\end{array}$ \\
\hline $\begin{array}{c}\text { Leucocitos } \\
>18.000 \mathrm{~m} 3\end{array}$ & BUN $>2 \mathrm{mg} / \mathrm{dL}$ \\
\hline $\begin{array}{c}\text { Glicemia }>220 \\
\mathrm{mg} / \mathrm{dL} \mid\end{array}$ & $\begin{array}{c}\text { Calcio sérico }<8 \\
\mathrm{mg} / \mathrm{dL}\end{array}$ \\
\hline $\begin{array}{c}\text { LDH sérica }>400 \\
\text { U/L }\end{array}$ & $\begin{array}{c}\text { Déficit de base }>5 \\
\mathrm{meq} / \mathrm{L}\end{array}$ \\
\hline AST $>250 \mathrm{U} / \mathrm{dL}$ & $\begin{array}{l}\text { Secuestro } \\
\text { estimado de } \\
\text { liquido }>4 \mathrm{~L}\end{array}$ \\
\hline
\end{tabular}

Tabla 7. Signos pronósticos de Ranson litiásica 


\section{CONCLUSIÓN}

Adquiere especial importancia el abordaje precoz y la aplicación correcta de las escalas para determinar la severidad del cuadro, además, tener en cuenta la posibilidad, ante el deterioro o la no mejoría clínica, de una disfunción multiorgánica que confiere una alta mortalidad. Con base en las actualizaciones más recientes de los ensayos clínicos, se evidencia la importancia de un manejo preferiblemente conservador $\mathrm{y}$, ante un cuadro más severo, de ser necesario, un manejo mínimamente invasivo.

\section{BIBLIOGRAFÍA}

1. Mullholland, M. 2017. Greenfield's Surgery Scientific Principles (6th edition) Wolters Kluwer.

2. Guidi M Curvale $C$ Pasqua $A$ et al. Actualización en el manejo inicial de la pancreatitis aguda. Acta Gastroenterol Latinoam 2019;49(4):307-323

3. Valdivieso Herrera, M., Vargas Ruiz, L., Arana Chiang, A., \& Piscoya, A. (2016). Situación epidemiológica de la pancreatitis aguda en Latinoamérica y alcances sobre el diagnóstico. Retrieved 26 May 2020, from https://www.redalyc.org/pdf/1993/199346231005.pdf

4. Forsmark, C., Swaroop Vege, S., \& Wilcox, M. (2016). Acute Pancreatitis. The New England Journal of Medicine. DOI: 10.1056/NEJMra1505202.

5. Losada Morales H, Troncoso Trujillo A, San Martín Ferrada P, Curitol Sánchez S. Comparación entre uso y no uso de antibióticos profilácticos en pancreatitis aguda grave. Ensayo Clínico Aleatorizado. Reporte preliminar. Rev. Cirugía. 2020;72(3). Disponible en: doi:10.35687/s245245492020003579

6. Álvarez Aguilar, P., \& Dobles Ramírez, C. (2019). Pancreatitis aguda: fisiopatología y manejo inicial. Retrieved 29 May 2020, from https://www.scielo.sa.cr/scielo.php?script=sci_ arttext\&pid=S0001-60022019000100013\#B19

7. Robbins LS, Cotran SR, Kumar V. Patología Estructural y Funcional. 10ma ed. España: Elsevier 2018.

8. Tenner, S., Baillie, J., DeWitt, J., \& Vege, S. S. (2013). American College of Gastroenterology Guideline: Management of Acute Pancreatitis. The American Journal of Gastroenterology, 108(9), 1400 -1415. doi:10.1038/ajg.2013.218

9. Guerrero, A., Flores de Miguel, Á., \&Alvillos, A. (2019). Pancreatitis aguda. Protocolo diagnóstico y terapéutico. doi:10.1016/j.med.2019.10.008

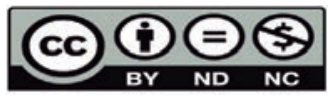


10. Ortiz Morales, C. M., Girela Baena, E. L., Olalla Muñoz, J. R., Parlorio de Andrés, E., \& López Corbalán, J. A. (2019). Radiología de la pancreatitis aguda hoy: clasificación de Atlanta y papel actual de la imagen en su diagnóstico y tratamiento. Radiología. doi:10.1016/j.rx.2019.04.001

11. Romero Urquhart, G. (2017). Acute Pancreatitis Imaging: Overview, Radiography, Computed Tomography. Retrieved 29 May 2020, from https://emedicine.medscape.com/article/371613overview

12. Boadas, J., Balsells, J., Busquets, J., Codina-B, A., Darnell, A., Garcia-Borobia, F., ... Molero, X. (2015). Valoración y tratamiento de la pancreatitis aguda. Documento de posicionamiento de la Societat Catalana de Digestologia, Societat Catalana de Cirurgia y Societat Catalana de Pàncrees. Gastroenterología y Hepatología, 38(2), 82-96. doi:10.1016/j.gastrohep.2014.09.006

13. Álvarez-López, F., Gómez-Cruz, Á., Cruz-Miranda, A., Martínez-Villaseñor, E., Alonzo-García, C., \& González-Álvarez, R. et al. (2020). Utilidad diagnóstica y pronóstica del tripsinógeno-2 urinario en pacientes con pancreatitis aguda. Retrieved 29 May 2020, from https://www. medigraphic.com/cgi-in/new/resumen.cgi?IDARTICULO=85141

14. Zertuche Cáceres, A., Calderón Du Pont, D., \& Hernandez Buen Abad, J. (2018). Análisis retrospectivo intrahospitalario del requerimiento de líquidos en pancreatitis aguda [Ebook] (63rd ed., pp. 169-175). Ciudad de Mexico: Anales Médicos de la Asociación Médica del Centro Médico ABC. Retrieved from http://www.medigraphic.com/analesmedicos

15. Hines, O., \& Pandol, S. (2019). Pancreatitis aguda grave. Retrieved 29 May 2020, from https:// www.intramed.net/contenidover.asp?contenidoid=95288\&pagina=1

16. Gómez Zuleta, M. A., Lúquez Mindiola, A. J., \& Ruíz Morales, O. F. (2017). Drenaje de pseudoquiste pancreático guiado por ecoendoscopia sin fluoroscopia: serie de casos. Revista Colombiana De Gastroenterología, 32(2), 160 - 165. https://doi.org/10.22516/25007440.143

17. Merino Rodríguez, E., Borobia Sánchez, R., Ramia Ángel, J. M., Rebolledo Olmedo, S., de la Plaza Llamas, R., \& Miquel Plaza, J. (2016). Fístula pancreático-colónica espontánea en paciente con pancreatitis aguda grave. Gastroenterología y Hepatología, 39(3), 221-223. doi:10.1016/j.gastrohep.2015.03.002 